\title{
Clinical Trial Expedited Safety Report
}

National Cancer Institute

\section{Source}

National Cancer Institute. Clinical Trial Expedited Safety Report. NCI Thesaurus. Code C115574.

Records describing relevant findings and notifications of unexpected serious adverse reactions and other safety information, which is submitted by a sponsor to regulatory authorities, investigators, and Institutional Review Boards (IRBs)/Independent Ethics Committees (IECs). 